\title{
HOMA insulin sensitivity index and the risk of all-cause mortality and cardiovascular disease events in the general population: the Australian Diabetes, Obesity and Lifestyle Study (AusDiab) study
}

\author{
E. L. M. Barr - A. J. Cameron - B. Balkau • \\ P. Z. Zimmet • T. A. Welborn • A. M. Tonkin • \\ J. E. Shaw
}

Received: 2 June 2009/Accepted: 24 September 2009/Published online: 7 November 2009

(C) Springer-Verlag 2009

\begin{abstract}
Aims/hypothesis We assessed whether the relationships between insulin sensitivity and all-cause mortality as well as fatal or non-fatal cardiovascular disease (CVD) events are independent of elevated blood glucose, high blood pressure, dyslipidaemia and body composition in individuals without diagnosed diabetes.

Methods Between 1999 and 2000, baseline fasting insulin, glucose and lipids, $2 \mathrm{~h}$ plasma glucose, $\mathrm{HbA}_{1 \mathrm{c}}$, anthropometrics, blood pressure, medication use, smoking and history
\end{abstract}

E. L. M. Barr $(\bowtie) \cdot$ A. J. Cameron · B. Balkau • P. Z. Zimmet •

J. E. Shaw

Department of Epidemiology and Clinical Diabetes,

Baker IDI Heart and Diabetes Institute,

250 Kooyong Road,

Caulfield 3162 VIC, Australia

e-mail: elizabeth.barr@bakeridi.edu.au

E. L. M. Barr • A. J. Cameron • P. Z. Zimmet • A. M. Tonkin •

J. E. Shaw

Department of Epidemiology and Preventive Medicine,

Monash University,

Melbourne, VIC, Australia

B. Balkau

INSERM U780,

Villejuif,

Paris, France

B. Balkau

University Paris-Sud,

Orsay,

Paris, France

T. A. Welborn

Department of Medicine, University of Western Australia,

Perth, WA, Australia of CVD were collected from 8,533 adults aged $>35$ years from the population-based Australian Diabetes, Obesity and Lifestyle study. Insulin sensitivity was estimated by HOMA of insulin sensitivity (HOMA-\%S). Deaths and fatal or nonfatal CVD events were ascertained through linkage to the National Death Index and medical records adjudication. Results After a median of 5.0 years there were 277 deaths and 225 CVD events. HOMA-\%S was not associated with all-cause mortality. Compared with the most insulinsensitive quintile, the combined fatal or non-fatal CVD HR $(95 \% \mathrm{CI})$ for quintiles of decreasing HOMA- $\% \mathrm{~S}$ were 1.1 (0.6-1.9), 1.4 (0.9-2.3), $1.6(1.0-2.5)$ and 2.0 (1.3-3.1), adjusting for age and sex. Smoking, CVD history, hypertension, lipid-lowering medication, total cholesterol and waist-to-hip ratio moderately attenuated this relationship. However, the association was rendered non-significant by adding HDL. Fasting plasma glucose, but not HOMA-\%S significantly improved the prediction of CVD, beyond that seen with other risk factors.

Conclusions/interpretation In this cohort, HOMA-\%S showed no association with all-cause mortality and only a modest association with CVD events, largely explained by its association with HDL. Fasting plasma glucose was a better predictor of CVD than HOMA-\%S.

Keywords Cardiovascular diseases · Hyperglycaemia . Insulin sensitivity and resistance $\cdot$ Metabolic syndrome . Mortality

Abbreviations

AusDiab Australian Diabetes, Obesity and Lifestyle

CVD Cardiovascular disease

FPG Fasting plasma glucose 


$\begin{array}{ll}\text { HOMA-IR } & \text { HOMA of insulin resistance } \\ \text { HOMA-\%S } & \text { HOMA of insulin sensitivity } \\ \text { 2hPG } & \text { 2 h plasma glucose } \\ \text { NDI } & \begin{array}{l}\text { National Death Index } \\ \text { PTCA }\end{array} \\ & \begin{array}{l}\text { Percutaneous transluminal coronary artery } \\ \text { angioplasty }\end{array}\end{array}$

\section{Introduction}

Insulin resistance precedes the development of hyperglycaemia and subsequent diabetes [1], which are both considered to be risk factors for the development of cardiovascular disease (CVD) and premature mortality [24]. Exploring the relative associations of hyperglycaemia and insulin resistance (or its reciprocal, insulin sensitivity) among persons without diagnosed diabetes may help elucidate possible mechanisms underlying premature mortality and CVD in the general population. Furthermore, as there is evidence of considerable heterogeneity in insulin resistance among individuals with different degrees of glucose tolerance [5], it is important to ascertain whether the presence of hyperglycaemia and insulin resistance can help identify high-risk groups.

In addition to hyperglycaemia, insulin resistance is also associated with several other CVD risk factors, including adiposity, dyslipidaemia and hypertension [6], which are collectively identified as the metabolic syndrome [7]. However, uncertainty remains as to whether insulin resistance is itself an independent risk factor for premature mortality and CVD. Although the euglycaemic-hyperinsulinaemic clamp is considered the gold standard method for measuring insulin resistance [8], few large studies have used it to evaluate the association between insulin resistance and CVD [9-11], as it is invasive and time-consuming. Consequently, surrogate markers of insulin resistance have been developed.

The simplest of these is plasma insulin, with two metaanalyses reporting a weak but statistically significant association between hyperinsulinaemia and CVD [12, 13]. However, one of these analyses combined results from several studies that did not adequately adjust for all components of the metabolic syndrome [12], while the other potentially introduced measurement error by combining individual level data from studies that had used different protocols and insulin assays (some specific and others not specific for insulin) [13].

Other markers of insulin resistance are based on both plasma glucose and insulin [14, 15]. The HOMA of insulin resistance (HOMA-IR) [16] and HOMA of insulin sensitivity (HOMA-\%S) [17] are commonly used to estimate insulin resistance and sensitivity, respectively. These models were developed from human and animal experimental data, with the updated HOMA- $\% \mathrm{~S}$ computer model being more accurate than the original HOMA-IR model [17]. Several [18-21], but not all [22-24] large prospective populationbased studies have found HOMA-IR values to be significantly associated with incident CVD, while the HOMA-\%S model values have been found to be associated with CVD in older women [25].

The Australian Diabetes, Obesity and Lifestyle (AusDiab) study is a national, prospective, population-based study of adult men and women, and represents the largest single study to assess the relationship between HOMA-\%S and CVD after adjusting for components of the metabolic syndrome, including blood glucose and other important CVD risk factors. In this study, we used insulin data from 8,533 men and women without diagnosed diabetes, as assessed with an insulinspecific assay, to investigate: (1) the associations between HOMA-\%S and both 5 year all-cause mortality rates and combined fatal or non-fatal CVD events; and (2) whether these relationships were independent of elevated blood glucose, high blood pressure, dyslipidaemia and body composition.

\section{Methods}

Study design The AusDiab study includes 11,247 men and women aged $\geq 25$ years. Methods and response rates are reported elsewhere [26, 27]. Between 1999 and 2000, a stratified clustered sample was drawn from 42 randomly selected census districts, six in each of the states and in the Northern Territory of Australia. The sampling frame consisted of 17,129 eligible households, from which 20,347 individuals completed a household interview. Of these, 11,247 (55\%) attended a biomedical examination, yielding an estimated response rate at baseline of $37 \%$. A 5 year follow-up study that included the assessment of nonfatal CVD outcomes was undertaken between 2004 and 2005 , and of the 10,788 eligible individuals, $8,802(82 \%)$ completed a questionnaire including history of CVD events. The Ethics Committees of the International Diabetes Institute, Monash University, the Australian Institute of Health and Welfare and the Sir Charles Gairdner Hospital, Western Australia approved the study, and informed consent was obtained from participants.

Baseline measures Baseline data on age, sex, use of antihypertensive and lipid-lowering medications, and history of both CVD (angina, myocardial infarction or stroke) and smoking were collected by interviewer-administered questionnaires. Measurements included blood pressure [28], anthropometrics [29] and a fasting ( $\geq 9 \mathrm{~h})$ blood sample. All participants, excluding pregnant women and people taking hypoglycaemic medication, underwent a 75 g OGTT. Fasting plasma insulin, fasting plasma glucose (FPG), $2 \mathrm{~h}$ plasma glucose $(2 \mathrm{hPG})$ and fasting serum total cholesterol, 
triacylglycerol and HDL were measured using an analyser (AU600; Olympus Optical, Tokyo, Japan). Glycated haemoglobin was measured from frozen samples of whole blood collected in EDTA tubes and stored at $-70^{\circ} \mathrm{C}$ for 2 to 36 months, using high-performance liquid chromatography (Bio-Rad Variant; Bio-Rad, Hercules, CA, USA) with standardised conversion to $\mathrm{HbA}_{1 \mathrm{c}}$ (normal range 4.2-6.3\%). Serum samples for insulin were stored at $-80^{\circ} \mathrm{C}$ until assayed. Serum insulin was measured using a human insulin-specific radioimmunoassay kit (Linco Research, St Charles, MO, USA). Insulin assays were only conducted in participants aged $>35$ years. All specimens were analysed at a central laboratory. Insulin sensitivity was estimated from FPG and fasting insulin concentrations using HOMA. HOMA-\%S was calculated with the HOMA-2 program [17].

Outcome measures Mortality status and both underlying and contributory causes of death were ascertained by linking all participants to the Australian National Death Index (NDI) [4]. People not matched to the NDI were assumed to be alive. The accuracy of the NDI for ascertainment of CVD deaths and vital status has been previously established [30]. Deaths were attributed to CVD if the underlying cause of death was coded I10-I25, I46.1, I48, I50-I99 or R96 according to the 2006 International Classification of Diseases 10th revision (www.who.int/ classifications/icd/en/, accessed 6 January 2009). In addition, participants with uncomplicated diabetes (E109, E119 or E149) or unspecified hyperlipidaemia (E785) as an underlying cause of death on the death certificate were attributed a CVD death $(n=3)$ if any of the CVD codes (I10-I25, I46.1, I48, I50-I99 or R96) were recorded in the first position on the death certificate.

During the 5 year follow-up study, 8,802 participants answered an interview-administered questionnaire on CVD events, including myocardial infarction, stroke, percutaneous transluminal coronary artery angioplasty (PTCA) and coronary artery bypass graft surgery. Self-reported CVD events that occurred between baseline and follow-up were adjudicated by two physicians (J. E. Shaw [diabetologist], A. M. Tonkin [cardiologist]) using medical records. This method has been validated against a state-wide hospital morbidity database [31]. A composite CVD outcome was defined as fatal or non-fatal myocardial infarction, fatal or non-fatal stroke, PTCA, coronary artery bypass graft or other deaths associated with CVD.

Participants were excluded if at baseline they: (1) were aged $\leq 35$ years $(n=1,603)$; (2) had known diabetes $(n=470)$ based on self-reported physician-diagnosed diabetes with use of hypoglycaemic medication or $\mathrm{FPG} \geq 7.0 \mathrm{mmol} / \mathrm{l}$ or $2 \mathrm{hPG} \geq 11.1 \mathrm{mmol} / \mathrm{l}$; (3) had not fasted for $\geq 9 \mathrm{~h}(n=16)$; (4) were pregnant $(n=17)$; (5) had FPG or $2 \mathrm{hPG}$ values $<2.5 \mathrm{mmol} / \mathrm{l}(n=37)$; and (6) had missing HOMA-\%S values $(n=40)$ or missing values for other covariates $(n=$ 527). Four participants who died but could not be matched to the NDI were excluded. Furthermore, 1,591 participants could not be included in the follow-up for fatal or non-fatal CVD events, as they did not complete the 2004-2005 nonfatal CVD questionnaire. Thus, for all-cause mortality the analyses were undertaken in 8,533 participants and for fatal or non-fatal CVD follow-up the analyses were based on 6,942 participants.

The follow-up period for all-cause mortality extended to the date of death or 7 April 2006, whichever came first. Follow-up of the composite fatal or non-fatal CVD events lasted until the date of event or death, or the individual's 2004-2005 testing date (between June 9, 2004 and April 7, 2006), whichever occurred first. CVD follow-up analyses were based on the first CVD event to occur after baseline testing. For people who died from CVD, non-fatal CVD events could not be obtained and therefore their CVD death was considered the CVD endpoint.

Statistical analysis To fully assess the relationship between HOMA-\%S and both all-cause mortality and CVD events, HOMA- $\% \mathrm{~S}$ was analysed as a continuous and categorical variable. For the continuous analysis, HOMA- $\% \mathrm{~S}$ had a positively skewed distribution and was log-transformed. Categories were based on quintiles of HOMA-\%S and were determined separately for all-cause mortality and CVD events follow-up. The first quintile represented individuals with the highest insulin sensitivity and the fifth quintile represented those with the lowest insulin sensitivity. Unadjusted mortality rates $(95 \% \mathrm{CI})$ per 1,000 person-years were calculated for each quintile of HOMA- $\%$ S. To test differences in means and proportions for baseline characteristics between the quintiles of HOMA-\%S, one-way analysis of variance and $\chi^{2}$ tests were used, respectively. FPG, $2 \mathrm{hPG}$ and triacylglycerol were not normally distributed and were logarithmically transformed prior to analysis.

Cox proportional hazards regression was used to examine the unadjusted and adjusted association between HOMA- $\% \mathrm{~S}$ and both all-cause mortality and CVD events (fatal or nonfatal). A linear or non-linear relationship between HOMA- $\% \mathrm{~S}$ and each of the outcomes was tested by calculating the likelihood ratio tests of a Cox model adjusted for age and sex, with and without the squared term for HOMA-\%S. Multivariate models were adjusted for age, sex, previous history of CVD (yes or no), smoking (never, ex-smoker or current smoker), hypertension (blood pressure $\geq 140 / 90 \mathrm{mmHg}$ or self-reported antihypertensive medication use, or blood pressure $<140 / 90 \mathrm{mmHg}$ and no medication use), systolic and diastolic blood pressure, total cholesterol, HDL, non-HDL (total cholesterol minus HDL), triacylglycerol, self-reported lipid-lowering medication use (yes or no), waist-to-hip ratio, waist circumference and BMI $\left(\mathrm{kg} / \mathrm{m}^{2}\right)$. Age, systolic and 
diastolic blood pressure, total cholesterol, HDL, non-HDL, triacylglycerol, waist-to-hip ratio, waist circumference and BMI were modelled as continuous variables. The influence of CVD risk factors on the relationship between HOMA-\%S and both all-cause mortality and CVD events was evaluated by: (1) entering each covariate individually after adjusting for age and sex to assess the impact of hypertension, dyslipidaemia, obesity and hyperglycaemia; and (2) building multivariate models to assess the combined effects of key covariates. Also, to assess whether the relationships between HOMA- $\% \mathrm{~S}$ and the outcome measures were independent of plasma glucose, HOMA- $\% \mathrm{~S}$ was modelled with FPG, $2 \mathrm{hPG}$ and $\mathrm{HbA}_{1 \mathrm{c}}$, adjusting for age, sex and the CVD covariates listed above. The predictive ability of HOMA- $\% \mathrm{~S}$ was compared with FPG, 2hPG and $\mathrm{HbA}_{1 \mathrm{c}}$ using log-likelihood ratio statistics, where a model containing HOMA-\%S, age, sex and CVD covariates was nested within models also containing FPG, $2 \mathrm{hPG}$ or $\mathrm{HbA}_{1 \mathrm{c}}$. For these models, $2 \mathrm{hPG}$ and $\mathrm{HbA}_{1 \mathrm{c}}$ were modelled as linear variables and FPG was modelled as a linear spline with one knot at $5.1 \mathrm{mmol} / \mathrm{l}$, as this functional form of FPG best describes the J-shaped relationship previously reported for FPG and CVD events [32].

Interactions between quintiles of HOMA- $\% \mathrm{~S}$ and sex, age ( $<65$ and $\geq 65$ years), previous history of CVD (no or yes), categories of abnormal glucose metabolism (normal glucose tolerance or impaired fasting glucose, impaired glucose tolerance or newly diagnosed diabetes), BMI ( $<25$ or $\geq 25$ to $<30$ or $\geq 30 \mathrm{~kg} / \mathrm{m}^{2}$ ) and waist-to-hip ratio (men: $\leq 0.95$ or $>0.95$, women: $\leq 0.80$ or $>0.80$ ) were tested by using log-likelihood ratio tests of models containing the variables as single terms nested within models including the first-order interactions. Interactions were considered significant at $p<0.01$.

All analyses were repeated using: (1) fasting insulin (pmol/l) as the surrogate measure of insulin sensitivity instead of HOMA- $\% \mathrm{~S}$; (2) a cohort that excluded individuals with newly diagnosed diabetes at baseline; and (3) a cohort that excluded individuals with self-reported myocardial infarction or stroke at baseline. Multi-collinearity between covariates was tested with the variance inflation factor, which was found to be $<3$ for all independent variables [33]. Proportional hazards assumptions were satisfied as assessed with graphs of log-log plots of the relative hazards by time for discrete variables and by scaled Schoenfeld residuals. Analyses were conducted with Stata Statistical Software version 9.2 (StataCorp, College Station, TX, USA).

\section{Results}

For the total cohort of 8,533 individuals the mean (range) age was $54(36-91)$ years, 4,700 (55\%) were women and
7,448 (87\%) reported being born in Australia, New Zealand or the UK. In unadjusted analyses (Table 1), declining insulin sensitivity, as measured by $\mathrm{HOMA}-\% \mathrm{~S}$, was significantly associated with other risk factors for premature mortality and CVD events.

There were 277 deaths (117 in women) after a median (interquartile range) of $5.0(0.5)$ years and 225 fatal or non-fatal CVD events after a median (interquartile range) of $5.0(0.2)$ years. Of these events, 74 were in women, 69 were fatal or nonfatal myocardial infarctions, 45 were fatal or non-fatal strokes, 45 were PTCAs and 35 were coronary artery bypass grafts; 31 were deaths due to other types of atherosclerotic CVD.

In unadjusted analyses, there was no relationship between HOMA-\%S and all-cause mortality (Fig. 1a). For CVD a linear association with HOMA-\%S was observed (Fig. 1b). Prediction of CVD was not improved by adding a squared term for HOMA-\%S ( $p>0.5$ in log-likelihood ratio test).

For all-cause mortality, no association was observed with quintiles of declining insulin sensitivity in either unadjusted (quintile HR [95\% CI]: 1.2 [0.8-1.8], 1.4 [1.0-2.1], 1.2 [0.8-1.8] and 1.3 [0.9-1.9]) or age- and sex-adjusted (quintile HR [95\% CI]: 1.1 [0.8-1.7], 1.0 [0.7-1.5], 0.9 [0.6-1.4] and 1.1 [0.7-1.6]) models. After adjustment for other covariates and re-analysing HOMA-\% $\%$ as a continuous variable, there was still no relationship with all-cause mortality (data not shown). Age, sex, waist-to-hip ratio, history of CVD and abnormal glucose tolerance status did not significantly modify the relationship between HOMA$\% \mathrm{~S}$ and all-cause mortality (all $p>0.03$ ).

In unadjusted analysis, individuals in the least insulinsensitive quintile of HOMA- $\% \mathrm{~S}$ were more than twice as likely to experience a CVD event than those in the most insulinsensitive quintile (Table 2). Adjustment for age, sex, glucose and other metabolic covariates only moderately influenced this relationship (Tables 2 and 3). Marked attenuation between HOMA-\%S and CVD was observed after adjusting for HDL ( $p=0.04$ for trend, age- and sex-adjusted [Table 2]; $p=0.14$ for trend, multivariate-adjusted [Table 3]).

Similar results were found when HOMA-\%S was modelled as a continuous variable. The fatal or non-fatal CVD HRs (95\% CI, $p$ ) per SD decrease of log-HOMA- $\%$ S were $1.36(1.20-1.54, p<0.01)$ in an unadjusted model and $1.30(1.14-1.49, p<0.01)$ after adjusting for age and sex. Although attenuated, the relationship remained significant after adjusting for previous history of CVD, smoking, hypertension (blood pressure $\geq 140 / 90 \mathrm{mmHg}$ or selfreported antihypertensive medication use), total cholesterol, self-reported lipid-lowering medication use and waist-tohip ratio $(1.24[1.08-1.43, p<0.01])$. However, the relationship became non-significant after further adjustment for HDL $(1.15[1.00-1.34, p=0.06])$.

FPG $(p<0.01)$, but not $2 \mathrm{hPG}(p=0.43)$ or $\mathrm{HbA}_{1 \mathrm{c}}(p=0.13)$ significantly improved the prediction of CVD after adjusting 
Table 1 Baseline characteristics according to quintiles of HOMA- $\% \mathrm{~S}$ in individuals without diagnosed diabetes $(n=8,533)$ : the AusDiab study

\begin{tabular}{|c|c|c|c|c|c|c|}
\hline \multirow[t]{2}{*}{ Baseline characteristics } & \multicolumn{5}{|c|}{ Values per quintiles of $\mathrm{HOMA}-\% \mathrm{~S}^{\mathrm{a}}$} & \multirow[t]{2}{*}{ Total } \\
\hline & 1 & 2 & 3 & 4 & 5 & \\
\hline HOMA-\%S & $>74.4$ & $74.4-59.1$ & $59.0-48.5$ & $48.4-36.9$ & $<36.8$ & $53(30)^{\mathrm{b}}$ \\
\hline Age, years & $52(12)$ & $54(12)$ & $56(13)$ & $56(12)$ & $55(12)$ & $54(12)$ \\
\hline Male, $n(\%)$ & $691(41)$ & $694(41)$ & $772(46)$ & $829(48)$ & $847(50)$ & $3,833(45)$ \\
\hline \multicolumn{7}{|l|}{ Smoking } \\
\hline Ex-smoker, $n(\%)$ & $478(28)$ & $493(29)$ & $500(30)$ & $550(32)$ & $583(34)$ & $2,604(31)$ \\
\hline Current smoker, $n(\%)$ & $323(19)$ & $256(15)$ & $231(14)$ & $206(12)$ & $220(13)$ & $1,236(15)$ \\
\hline History of CVD, $n(\%)^{\mathrm{c}}$ & $110(6)$ & $113(7)$ & $153(9)$ & $165(10)$ & $177(10)$ & $718(8)$ \\
\hline Hypertension, $n(\%)^{\mathrm{d}}$ & $373(22)$ & $457(27)$ & $586(35)$ & $708(41)$ & $883(52)$ & $3,007(35)$ \\
\hline Systolic blood pressure $(\mathrm{mmHg})$ & $124(18)$ & $127(18)$ & $131(18)$ & $133(19)$ & $137(18)$ & $131(19)$ \\
\hline Diastolic blood pressure $(\mathrm{mmHg})$ & $67(12)$ & $69(12)$ & $71(11)$ & $72(12)$ & $75(12)$ & $71(12)$ \\
\hline Total cholesterol $(\mathrm{mmol} / \mathrm{l})$ & $5.5(1.1)$ & $5.7(1.0)$ & $5.8(1.0)$ & $5.9(1.1)$ & $5.9(1.1)$ & $5.8(1.1)$ \\
\hline HDL (mmol/l) & $1.6(0.4)$ & $1.6(0.4)$ & $1.5(0.4)$ & $1.4(0.3)$ & $1.2(0.3)$ & $1.4(0.4)$ \\
\hline Triacylglycerol $(\mathrm{mmol} / \mathrm{l})^{\mathrm{b}}$ & $1.0(0.6)$ & $1.1(0.8)$ & $1.3(0.9)$ & $1.5(1.1)$ & $1.9(1.3)$ & $1.3(1.0)$ \\
\hline Lipid-lowering medication use, $n(\%)$ & $74(4)$ & $122(7)$ & $166(10)$ & $174(10)$ & $214(13)$ & $750(9)$ \\
\hline Waist-to-hip ratio & $0.83(0.8)$ & $0.84(0.8)$ & $0.90(0.8)$ & $0.90(0.9)$ & $0.92(0.8)$ & $0.90(0.9)$ \\
\hline BMI $\left(\mathrm{kg} / \mathrm{m}^{2}\right)$ & $24(4)$ & $25(4)$ & $27(4)$ & $28(4)$ & $31(5)$ & $27(5)$ \\
\hline $\mathrm{FPG}(\mathrm{mmol} / \mathrm{l})^{\mathrm{b}}$ & $5.1(0.5)$ & $5.3(0.6)$ & $5.4(0.6)$ & $5.5(0.6)$ & $5.8(0.9)$ & $5.4(0.7)$ \\
\hline $2 \mathrm{hPG}(\mathrm{mmol} / \mathrm{l})^{\mathrm{b}}$ & $5.4(1.9)$ & $5.6(1.9)$ & $5.8(2.0)$ & $6.2(2.1)$ & $7.0(2.9)$ & $5.9(2.2)$ \\
\hline $\mathrm{HbA}_{1 \mathrm{c}}(\%)^{\mathrm{b}}$ & $5.0(0.3)$ & $5.0(0.3)$ & $5.1(0.3)$ & $5.2(0.4)$ & $5.3(0.4)$ & $5.1(0.4)$ \\
\hline NGT, $n(\%)$ & $1,480(87)$ & $1,442(84)$ & $1,331(79)$ & $1,211(70)$ & $842(49)$ & $6,306(74)$ \\
\hline IFG, $n(\%)$ & $38(2)$ & $64(4)$ & 107 (6) & $151(9)$ & $212(12)$ & $572(7)$ \\
\hline IGT, $n(\%)$ & $154(9)$ & $175(10)$ & $205(12)$ & 294 (17) & $415(24)$ & $1,243(15)$ \\
\hline NDM, $n(\%)$ & $29(2)$ & $31(2)$ & $45(3)$ & $67(4)$ & $240(14)$ & $412(5)$ \\
\hline
\end{tabular}

Data are $n(\%)$ or mean $(\mathrm{SD})$

${ }^{\mathrm{a}}$ Increasing quintiles of $\mathrm{HOMA}-\% \mathrm{~S}$ represent decreasing insulin sensitivity

${ }^{\mathrm{b}}$ Data are median (interquartile range)

${ }^{\mathrm{c}}$ History of CVD includes self-reported angina, myocardial infarction or stroke

${ }^{\mathrm{d}}$ Defined as blood pressure $\geq 140 / 90 \mathrm{mmHg}$ or self-reported antihypertensive medication use

Differences across quintiles of HOMA-\%S were significant for all variables $(p<0.01)$

IFG, impaired fasting glucose; IGT, impaired glucose tolerance; NDM, newly diagnosed diabetes; NGT, normal glucose tolerance

for $\mathrm{HOMA}-\% \mathrm{~S}$ and the covariates (Table 4). Adding quintiles of HOMA-\%S to multivariate models with FPG, $2 \mathrm{hPG}$ or $\mathrm{HbA}_{1 \mathrm{c}}$ did not improve the prediction of CVD $(p>$ 0.6 for all). Multivariate models including HOMA- $\% \mathrm{~S}$ and
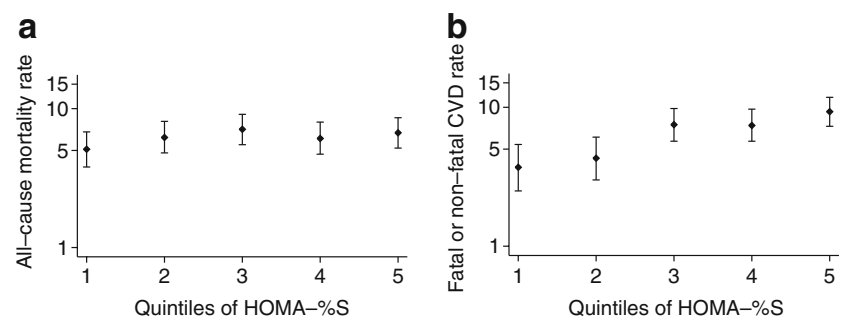

Fig. 1 Unadjusted rates per 1,000 person-years for all-cause mortality (a) and fatal or non-fatal CVD events (b) according to quintiles of decreasing HOMA- $\% \mathrm{~S}$ in individuals without diagnosed diabetes: the AusDiab study. $y$-axes in $\log _{10}$ scale plasma glucose measures showed that the association between CVD and FPG was of borderline significance (HR $3.6[95 \%$ CI $1.5-8.5, p<0.01]$ per SD $[0.7 \mathrm{mmol} / \mathrm{l}]$ decrease for $\mathrm{FPG}<5.1 \mathrm{mmol} / 1$ and $1.1[1.0-1.2, p=0.05]$ per $\mathrm{SD}$ increase for $\mathrm{FPG} \geq 5.1 \mathrm{mmol} / \mathrm{l}$ ), whereas $2 \mathrm{hPG}$ and $\mathrm{HbA}_{1 \mathrm{c}}$ were not significantly associated with CVD when modelled with HOMA-\%S (HR 1.0 [95\% CI 0.9-1.2, $p=0.42]$ per SD [2.2 mmol/1] increase for 2hPG; HR 1.1 [95\% CI 1.0-1.2, $p=$ $0.10]$ per SD [0.4\%] increase for $\mathrm{HbA}_{1 \mathrm{c}}$ ).

No significant interactions were observed between HOMA-\%S (in quintiles) and age, sex, BMI, waist-to-hip ratio, history of CVD or abnormal glucose tolerance status for CVD (all $p>0.05$ ). For individuals without a selfreported history of myocardial infarction or stroke at baseline $(n=6,590)$, the HRs $(95 \% \mathrm{CI})$ for quintiles of decreasing HOMA-\%S were 0.8 (0.4-1.4), 1.2 (0.7-2.1), 
Table 2 Unadjusted and age- and sex-adjusted risk of fatal or non-fatal CVD events according to quintiles of HOMA-\%S in individuals without diagnosed diabetes $(n=6,942)$ : the AusDiab study

\begin{tabular}{|c|c|c|c|c|c|c|}
\hline \multirow[t]{2}{*}{ Variables } & \multicolumn{5}{|c|}{ Values per quintiles of $\mathrm{HOMA}-\% \mathrm{~S}^{\mathrm{a}}$} & \multirow[t]{2}{*}{$p$ value for tren } \\
\hline & 1 & 2 & 3 & 4 & 5 & \\
\hline HOMA-\%S & $>74.6$ & $74.6-59.3$ & $59.2-48.7$ & $48.6-37.2$ & $<37.1$ & \\
\hline CVD events $n(\%)$ & $26(2.0)$ & $30(2.2)$ & $52(3.8)$ & $52(3.7)$ & $65(4.7)$ & \\
\hline \multicolumn{7}{|l|}{ Risk in HR $(95 \% \mathrm{CI})$} \\
\hline Unadjusted & 1.0 & $1.2(0.7-2.0)$ & $2.0(1.3-3.2)^{*}$ & $2.0(1.3-3.2)^{*}$ & $2.5(1.6-3.9)^{*}$ & $<0.01$ \\
\hline \multicolumn{7}{|l|}{ Adjusted for } \\
\hline Age + sex & 1.0 & $1.1(0.6-1.9)$ & $1.4(0.9-2.3)$ & $1.6(1.0-2.5)$ & $2.0(1.3-3.1)^{*}$ & $<0.01$ \\
\hline $\mathrm{Age}+\mathrm{sex}+\mathrm{FPG}^{\mathrm{c}}$ & 1.0 & $1.1(0.7-2.0)$ & $1.5(0.9-2.4)$ & $1.6(1.0-2.7)^{*}$ & $2.0(1.2-3.2)^{*}$ & $<0.01$ \\
\hline Age + sex $+2 h P^{c, d}$ & 1.0 & $1.1(0.6-1.8)$ & $1.4(0.9-2.3)$ & $1.5(0.9-2.4)$ & $1.9(1.2-2.9)^{*}$ & $<0.01$ \\
\hline $\mathrm{Age}+\mathrm{sex}+\mathrm{HbA}_{1 \mathrm{c}}{ }^{\mathrm{c}}$ & 1.0 & $1.1(0.6-1.8)$ & $1.4(0.9-2.2)$ & $1.5(0.9-2.4)$ & $1.8(1.1-2.8)^{*}$ & $<0.01$ \\
\hline Age + sex + history of CVD & 1.0 & $1.2(0.7-2.0)$ & $1.6(1.0-2.5)$ & $1.6(1.0-2.6)^{*}$ & $2.1(1.3-3.3)^{*}$ & $<0.01$ \\
\hline Age + sex + smoking & 1.0 & $1.1(0.7-1.9)$ & $1.5(0.9-2.3)$ & $1.6(1.0-2.5)^{*}$ & $2.0(1.3-3.2)^{*}$ & $<0.01$ \\
\hline Age + sex + hypertension & 1.0 & $1.1(0.6-1.8)$ & $1.4(0.9-2.2)$ & $1.5(0.9-2.3)$ & $1.7(1.1-2.8)^{*}$ & 0.01 \\
\hline Age + sex + SBP and DBP & 1.0 & $1.1(0.6-1.8)$ & $1.4(0.9-2.2)$ & $1.5(0.9-2.4)$ & $1.8(1.1-2.8)^{*}$ & $<0.01$ \\
\hline Age + sex + lipid-lowering therapy and TC & 1.0 & $1.1(0.6-1.8)$ & $1.4(0.8-2.2)$ & $1.5(0.9-2.4)$ & $1.9(1.2-2.9)^{*}$ & $<0.01$ \\
\hline Age + sex + triacylglycerol & 1.0 & $1.1(0.6-1.8)$ & $1.4(0.9-2.2)$ & $1.4(0.9-2.3)$ & $1.7(1.1-2.8)^{*}$ & 0.01 \\
\hline Age + sex + non-HDL & 1.0 & $1.1(0.6-1.8)$ & $1.4(0.9-2.3)$ & $1.5(0.9-2.4)$ & $1.9(1.2-3.0)^{*}$ & $<0.01$ \\
\hline $\mathrm{Age}+\mathrm{sex}+\mathrm{HDL}$ & 1.0 & $1.0(0.6-1.8)$ & $1.3(0.8-2.1)$ & $1.3(0.8-2.1)$ & $1.5(1.0-2.5)$ & 0.04 \\
\hline Age + sex + WHR & 1.0 & $1.1(0.6-1.8)$ & $1.4(0.9-2.3)$ & $1.5(0.9-2.4)$ & $1.9(1.2-3.0)^{*}$ & $<0.01$ \\
\hline Age + sex + waist circumference & 1.0 & $1.1(0.6-1.8)$ & $1.4(0.9-2.3)$ & $1.5(0.9-2.5)$ & $1.9(1.2-3.1)^{*}$ & $<0.01$ \\
\hline $\mathrm{Age}+\mathrm{sex}+\mathrm{BMI}$ & 1.0 & $1.1(0.6-1.8)$ & $1.4(0.9-2.3)$ & $1.5(0.9-2.4)$ & $1.9(1.1-3.1)^{*}$ & 0.01 \\
\hline
\end{tabular}

${ }^{\mathrm{a}}$ Increasing quintiles of $\mathrm{HOMA}-\% \mathrm{~S}$ represent decreasing insulin sensitivity

${ }^{\mathrm{b}}$ Total CVD events: 225 (3.2)

${ }^{\mathrm{c}} \mathrm{FPG}$ was modelled as a linear spline with a knot at $5.1 \mathrm{mmol} / \mathrm{l}$; $\mathrm{HbA}_{1 \mathrm{c}}$ and $2 \mathrm{hPG}$ modelled as linear terms

${ }^{\mathrm{d}}$ Models based on 6,933 participants with non-missing $2 \mathrm{hPG}$ values. Quintiles based on this sub-cohort

* $p<0.05$

DPB, diastolic BP; SBP, systolic BP; TC, total cholesterol

$1.1(0.7-1.9)$ and $1.7(1.1-2.9)(p<0.01$ for trend $)$ after adjusting for age and sex. For individuals with a selfreported history of myocardial infarction or stroke at baseline $(n=352)$, the corresponding HRs $(95 \% \mathrm{CI})$ were $2.5(0.9-7.2), 3.3(1.2-9.3), 3.5(1.3-9.7), 2.6(0.9-7.6)$ ( $p=0.06$ for trend). Results were essentially unchanged when we repeated these analyses excluding people with newly diagnosed diabetes or replacing HOMA-\%S with fasting insulin. Adding education level or physical activity did not alter the association between HOMA- $\% \mathrm{~S}$ and CVD (data not shown).

\section{Discussion}

In this population-based prospective study of men and women we examined the relationship between HOMA-\%S and both CVD and all-cause mortality, after accounting for a broad range of CVD risk factors, including previous history of CVD, smoking, hypertension, total cholesterol, lipidlowering medication use, dyslipidaemia and obesity. We found that declining insulin sensitivity was associated with 5 year fatal or non-fatal CVD events, but not with all-cause mortality. The relationship between HOMA-\%S and CVD events was largely explained by HDL, which significantly attenuated the association between HOMA-\%S and CVD. Furthermore, only FPG significantly improved the prediction of CVD beyond that achieved by HOMA-\%S and other CVD risk factors. There were no significant interactions between HOMA- $\% \mathrm{~S}$ and age, sex, glucose tolerance categories, history of CVD or obesity.

The question of whether insulin resistance directly leads to CVD or whether it has an indirect deleterious effect via other metabolic abnormalities is subject to debate. Findings from studies using a direct measurement of insulin sensitivity are limited. Although decreasing insulin sensitivity has been shown to be independently associated with an increased risk of CVD [9, 10], one of the studies only 
Table 3 Multivariate adjusted risk of fatal or non-fatal CVD events according to quintiles of HOMA-\%S in individuals without diagnosed diabetes $(n=6,942)$ : the AusDiab study

\begin{tabular}{|c|c|c|c|c|c|c|}
\hline \multirow[t]{2}{*}{ Variables } & \multicolumn{5}{|c|}{ Values per quintiles of $\mathrm{HOMA}-\% \mathrm{~S}^{\mathrm{a}}$} & \multirow[t]{2}{*}{$p$ value for trend } \\
\hline & 1 & 2 & 3 & 4 & 5 & \\
\hline HOMA-\%S & $>74.6$ & $74.6-59.3$ & $59.2-48.7$ & $48.6-37.2$ & $<37.1$ & \\
\hline CVD events, $n(\%)^{\mathrm{b}}$ & $26(2.0)$ & $30(2.2)$ & $52(3.8)$ & $52(3.7)$ & $65(4.7)$ & \\
\hline $\begin{array}{l}\text { Risk (HR }[95 \% \mathrm{CI}] \text { ) adjusted } \\
\text { for age, sex, history of } \mathrm{CVD}^{\mathrm{c}} \\
\text { and smoking }\end{array}$ & 1.0 & $1.2(0.7-2.0)$ & $1.6(1.0-2.5)$ & $1.6(1.0-2.6)^{*}$ & $2.1(1.3-3.3)^{*}$ & $<0.01$ \\
\hline Plus hypertension & 1.0 & $1.2(0.7-2.1)$ & $1.5(0.9-2.4)$ & $1.6(1.0-2.5)$ & $1.9(1.2-3.0)^{*}$ & $<0.01$ \\
\hline Plus WHR & 1.0 & $1.2(0.7-2.1)$ & $1.5(0.9-2.4)$ & $1.5(1.0-2.5)$ & $1.9(1.2-3.0)^{*}$ & 0.01 \\
\hline Plus LLT and TC & 1.0 & $1.2(0.7-2.0)$ & $1.4(0.9-2.3)$ & $1.5(0.9-2.4)$ & $1.8(1.1-2.9)^{*}$ & 0.01 \\
\hline Plus hypertension $+\mathrm{LLT}$ and $\mathrm{TC}+\mathrm{TG}$ & 1.0 & $1.2(0.7-2.0)$ & $1.4(0.9-2.3)$ & $1.5(0.9-2.4)$ & $1.7(1.1-2.8)^{*}$ & 0.01 \\
\hline Plus non-HDL ${ }^{\mathrm{d}}$ & 1.0 & $1.2(0.7-2.0)$ & $1.4(0.9-2.2)$ & $1.5(0.9-2.3)$ & $1.7(1.1-2.7)^{*}$ & 0.01 \\
\hline Plus HDL & 1.0 & $1.1(0.7-1.9)$ & $1.3(0.8-2.1)$ & $1.3(0.8-2.1)$ & $1.4(0.9-2.3)$ & 0.14 \\
\hline $\begin{array}{l}\text { Plus hypertension+LLT and } \\
\text { TC+HDL+WHR }\end{array}$ & 1.0 & $1.2(0.7-2.0)$ & $1.3(0.8-2.1)$ & $1.3(0.8-2.2)$ & $1.5(0.9-2.4)$ & 0.12 \\
\hline
\end{tabular}

${ }^{\mathrm{a}}$ Increasing quintiles of HOMA-\%S represent decreasing insulin sensitivity

${ }^{\mathrm{b}}$ Total CVD events: 225 (3.2)

${ }^{\mathrm{c}}$ History of previous CVD includes self-reported angina, myocardial infarction or stroke

${ }^{\mathrm{d}}$ Model does not include total cholesterol, as non-HDL and total cholesterol are highly correlated $(r=0.93)$

* $p<0.05$

LLT, lipid-lowering medication use; TC, total cholesterol; TG, triacylglycerol

used a surrogate CVD outcome (carotid artery intimamedia thickness) and evaluated insulin resistance with an intravenous glucose tolerance test [9], while the other study (using the insulin clamp) only involved older men [10].
Several prospective studies using surrogate measures of insulin resistance have shown that the metabolic syndrome significantly attenuates the relationship between HOMA-IR and CVD [22-24], supporting the notion of an indirect link

Table 4 Risk of fatal or non-fatal CVD events according to quintiles of HOMA- $\% \mathrm{~S}$ and adjusted for FPG, $2 \mathrm{hPG}$ and $\mathrm{Hb} \mathrm{A}_{1 \mathrm{c}}$ in individuals without diagnosed diabetes $(n=6,942)$ : the AusDiab study

\begin{tabular}{|c|c|c|c|c|c|c|c|c|c|c|}
\hline \multirow[t]{3}{*}{$\begin{array}{l}\text { HOMA-\%S } \\
\text { quintiles }^{\mathrm{a}}\end{array}$} & \multirow{3}{*}{$\begin{array}{l}\text { Multivariate-adjusted }^{\mathrm{b}} \\
\text { HR }(95 \% \mathrm{CI})\end{array}$} & \multicolumn{3}{|c|}{$\begin{array}{l}\text { Additionally adjusted } \\
\text { for } \mathrm{FPG}^{\mathrm{b}, \mathrm{c}}\end{array}$} & \multicolumn{3}{|c|}{$\begin{array}{l}\text { Additionally adjusted } \\
\text { for } 2 \mathrm{hPG}^{\mathrm{b}} \text {, d, e }\end{array}$} & \multicolumn{3}{|c|}{$\begin{array}{l}\text { Additionally adjusted } \\
\text { for } \mathrm{HbA}_{1 \mathrm{c}} \mathrm{b}, \mathrm{e}\end{array}$} \\
\hline & & \multirow[t]{2}{*}{ HR (95\% CI) } & \multicolumn{2}{|c|}{$\begin{array}{l}\text { Likelihood } \\
\text { ratio test }\end{array}$} & \multirow[t]{2}{*}{ HR $(95 \%$ CI) } & \multicolumn{2}{|c|}{$\begin{array}{l}\text { Likelihood } \\
\text { ratio test }\end{array}$} & \multirow[t]{2}{*}{ HR $(95 \%$ CI) } & \multicolumn{2}{|c|}{$\begin{array}{l}\text { Likelihood } \\
\text { ratio test }\end{array}$} \\
\hline & & & $\chi^{2}$ & $p$ value & & $\chi^{2}$ & $p$ value & & $\chi^{2}$ & $p$ value \\
\hline$>74.6$ & 1.0 & 1.0 & 11.8 & $<0.01$ & 1.0 & 0.64 & 0.43 & 1.0 & 2.34 & 0.13 \\
\hline $74.6-59.3$ & $1.2(0.7-2.0)$ & $1.2(0.7-2.0)$ & & & $1.1(0.7-1.9)$ & & & $1.1(0.7-1.9)$ & & \\
\hline $59.2-48.7$ & $1.3(0.8-2.1)$ & $1.4(0.9-2.2)$ & & & $1.3(0.8-2.1)$ & & & $1.3(0.8-2.1)$ & & \\
\hline $48.6-37.2$ & $1.3(0.8-2.2)$ & $1.4(0.9-2.3)$ & & & $1.3(0.8-2.1)$ & & & $1.3(0.8-2.1)$ & & \\
\hline$<37.1$ & $1.5(0.9-2.4)$ & $1.5(0.9-2.5)$ & & & $1.4(0.9-2.4)$ & & & $1.4(0.8-2.3)$ & & \\
\hline$p$ value for trend & 0.12 & 0.11 & & & 0.15 & & & 0.21 & & \\
\hline
\end{tabular}

${ }^{\mathrm{a}}$ Increasing quintiles of HOMA-\%S represent decreasing insulin sensitivity

${ }^{\mathrm{b}}$ Adjusted for age, sex, history of CVD (angina, myocardial infarction or stroke), smoking (never smoked, ex-smoker, current smoker), hypertension (blood pressure $\geq 140 / 90 \mathrm{mmHg}$ or self-reported antihypertensive use), total cholesterol, HDL-cholesterol, lipid-lowering medication use and waist-to-hip ratio

${ }^{\mathrm{c}}$ FPG added to model as a linear spline with one knot at $5.1 \mathrm{mmol} / \mathrm{l}$

${ }^{\mathrm{d}}$ Models based on 6,933 participants with non-missing $2 \mathrm{hPG}$ values. Quintiles based on this sub-cohort

e $2 \mathrm{hPG}$ and $\mathrm{HbA}_{1 \mathrm{c}}$ added to multivariate adjusted model as continuous variables 
between insulin resistance and CVD through other metabolic pathways [34]. However, it is not possible to determine from these studies which metabolic factor had the strongest influence, as the metabolic syndrome was only considered in its entirety. Few epidemiological investigations have assessed the relative impact of several metabolic risk factors on the association between insulin resistance and CVD [19, 21], but those that did used HOMA-IR and not the updated HOMA-\%S model. Our findings extend this previous work $[19,21]$, because we have specifically examined the individual impact of several metabolic risk factors and used HOMA-\%S as the measure of insulin sensitivity.

The findings from our study seem to support the lipotoxicity hypothesis and suggest that HDL may be an important confounder of the association between insulin resistance and CVD. Although insulin resistance and dyslipidaemia (high triacylglycerol, low HDL) are characteristic of type 2 diabetes [35], individuals with diagnosed diabetes were excluded in this analysis, suggesting that insulin resistance and dyslipidaemia may also play a role in the development of CVD in the general population. The disappearance of the association between HOMA- $\% \mathrm{~S}$ and CVD after adjustment for HDL suggests that either: (1) HDL is the key risk factor (and that the univariate association between HOMA- $\% \mathrm{~S}$ and CVD is due to the links between insulin sensitivity and HDL levels); or (2) low HDL is a consequence of insulin resistance and that adjusting for it is potentially an over-adjustment. However, our data do not allow us to assess which of the above applies. It is also possible that other underlying factors are responsible for the development of insulin resistance and abnormal HDL metabolism. One theory suggests that obesity may be a common antecedent, as it leads to an increase in circulating NEFA, which in turn have a deleterious effect on insulin resistance and associated hepatic lipoprotein abnormalities, including a reduction in HDL [36]. Indeed, waist circumference has been shown to be more strongly associated with and to precede the development of metabolic abnormalities than insulin resistance measured by HOMA-\%S [37]. However, it was not possible to evaluate this further in this study.

Nevertheless, other studies have shown HOMA-IR [19, $21]$ and hyperinsulinaemia $[13,25,38]$ to be significantly associated with CVD, despite adjusting for HDL and other metabolic factors. However, all but two [25, 38] of these studies used a non-specific insulin assay which cross-reacts with proinsulin $[13,19,21]$. As proinsulin may be a better predictor of CVD than fasting insulin [10] or HOMA-IR [39], the association between true insulin and CVD may have been concealed in these previous studies. Moreover, the findings from the two studies that did use a specific insulin assay may not be generalisable to the general population as one was conducted in older women [25] and the other only included men and measured non-fasting insulin [38].

Many studies have reported on the CVD risks associated with non-diabetic hyperglycaemia and insulin resistance, but few have compared the relative associations between these conditions and CVD in the same study population, and results are inconsistent. Several studies [40-42], including a meta-analysis [13], have reported a significant association between hyperinsulinaemia and CVD events after adjusting for glucose. However, these associations may have been the result of residual confounding, as none adjusted for HDL or waist circumference, factors that are known to coexist with insulin resistance and also to increase the likelihood of CVD [7]. Furthermore, although some studies have found blood glucose to be a better predictor of CVD than hyperinsulinaemia [43] or HOMAIR [24], others have not $[25,38]$. However, the latter two studies were conducted in particular populations: older women [25] and middle-aged men [38]. In our study, prediction of CVD events significantly improved with the addition of FPG, but not HOMA- $\% \mathrm{~S}$ to models adjusted for age, sex and other CVD risk factors. This suggests that in the general population, FPG and other CVD risk factors, rather than HOMA- $\% \mathrm{~S}$, may be the important targets for prevention of CVD.

Several limitations need to be considered when interpreting these results. First, as this is a large populationbased study, it was not feasible to measure insulin sensitivity directly with an insulin clamp. Instead, insulin sensitivity was estimated with HOMA-\%S, which is a relatively simplistic index of insulin sensitivity calculated from fasting plasma insulin and glucose levels. Consequently, the non-significant findings in this study may be attributable to the imprecision of HOMA-\%S. Although HOMA-\%S has been found to be significantly correlated $(r=0.88)$ with insulin sensitivity, this finding was only based on a small study using the minimal model [44]. Thus, further research using the insulin clamp is required to confirm our findings. Second, we were not able to account for the influence of intra-individual variation in HOMA- $\% \mathrm{~S}$ measurements, as they were based on only one measurement. However, findings from large datasets have shown that measurements using a single sample yield similar results to those from multiple samples [17]. Third, this study examined the risk of CVD and mortality over a 5 year period, which may not be long enough to detect a significant association, although several other studies have demonstrated that the relationship between insulin resistance (as estimated with hyperinsulinaemia) and CVD becomes weaker with time [45]. Finally, the generalisability of this study to the Australian population is limited by the estimated baseline response rate of $37 \%$. Non- 
English speaking Australians and people who completed less than 12 years of school were under-represented in AusDiab, so these findings may not be generalisable to these groups [26].

In conclusion, this prospective population-based study of men and women evaluated the association between HOMA-\%S and CVD after adjusting for a wide range of CVD risk factors. It is also one of the few studies to use a specific insulin assay to measure serum insulin levels. We found FPG to be a better predictor of CVD than HOMA$\% \mathrm{~S}$. Over a 5 year period there was no association between HOMA- $\% \mathrm{~S}$ and all-cause mortality, and only a modest relationship between HOMA- $\% \mathrm{~S}$ and both fatal or non-fatal CVD events, mainly explained by the clustering of other risk factors, in particular HDL. These findings are consistent with the notion that insulin resistance plays an indirect role in the development of CVD, although the question of whether insulin resistance is the cause of other metabolic abnormalities remains debatable and requires further research.

Acknowledgements $\quad$ E. L. M. Barr is supported by a National Health and Medical Research Council (NHMRC; 379305)/National Heart Foundation Australia (PP 05M 2346) joint postgraduate scholarship. A. J. Cameron is supported by Postgraduate Research Scholarship (PP 04M 1794) from the National Heart Foundation of Australia. The AusDiab study, co-coordinated by the Baker Heart and Diabetes Research Institute, gratefully acknowledges the generous support given by: National Health and Medical Research Council (NHMRC grant 233200), National Heart Foundation Australia (Research Grantin-Aid RES17-01 2005) for the collection of and adjudication of medical records and in-kind support from the Australian Institute of Health and Welfare, who provided the mortality data. In addition, we are most grateful to the following for their support: The Commonwealth Department of Health and Aged Care, Abbott Australasia, Alphapharm, AstraZeneca, Aventis Pharmaceutical, Bristol-Myers Squibb Pharmaceuticals, Eli Lilly (Australia), GlaxoSmithKline, Janssen-Cilag (Australia), Merck Lipha, Merck Sharp \& Dohme (Australia), Novartis Pharmaceutical (Australia), Novo Nordisk Pharmaceutical, Pharmacia and Upjohn, Pfizer, Roche Diagnostics, Sanofi Synthelabo (Australia), Servier Laboratories (Australia), BioRad Laboratories, HITECH Pathology, the Australian Kidney Foundation, Diabetes Australia, Diabetes Australia (Northern Territory), Queensland Health, South Australian Department of Human Services, Tasmanian Department of Health and Human Services, Territory Health Services and Victorian Department of Human Services, and Health Department of Western Australia. For their invaluable contribution to the set-up and field activities of AusDiab, we are enormously grateful to: A. Allman, B. Atkins, S. Bennett, A. Bonney, S. Chadban, M. de Courten, M. Dalton, D. Dunstan, T. Dwyer, H. Jahangir, D. Jolley, D. McCarty, A. Meehan, N. Meinig, S. Murray, K. O'Dea, K. Polkinghorne, P. Phillips, C. Reid, A. Stewart, R. Tapp, H. Taylor, T. Whalen and F. Wilson. A special acknowledgement also goes to S. Murray, G. Filby and medical records staff, who helped in the collection of medical record information. Finally, we thank the AusDiab participants for volunteering their time to participate in the study.

Duality of interest The authors declare that there is no duality of interest associated with this manuscript.

\section{References}

1. Kahn SE (2003) The relative contributions of insulin resistance and beta-cell dysfunction to the pathophysiology of type 2 diabetes. Diabetologia 46:3-19

2. Coutinho M, Gerstein HC, Wang Y, Yusuf S (1999) The relationship between glucose and incident cardiovascular events. A metaregression analysis of published data from 20 studies of 95 , 783 individuals followed for 12.4 years. Diabetes Care 22:233-240

3. The DECODE study group on behalf of the European Diabetes Epidemiology Group (2001) Glucose tolerance and cardiovascular mortality: comparison of fasting and 2-hour diagnostic criteria. Arch Intern Med 161:397-405

4. Barr EL, Zimmet PZ, Welborn TA et al (2007) Risk of cardiovascular and all-cause mortality in individuals with diabetes mellitus, impaired fasting glucose, and impaired glucose tolerance: the Australian Diabetes, Obesity, and Lifestyle Study (AusDiab). Circulation 116:151-157

5. Kim SH, Reaven GM (2008) Isolated impaired fasting glucose and peripheral insulin sensitivity: not a simple relationship. Diabetes Care 31:347-352

6. Ferrannini E, Haffner SM, Mitchell BD, Stern MP (1991) Hyperinsulinaemia: the key feature of a cardiovascular and metabolic syndrome. Diabetologia 34:416-422

7. Reaven GM (1988) Banting lecture 1988. Role of insulin resistance in human disease. Diabetes 37:1595-1607

8. American Diabetes Association (1998) Consensus Development Conference on Insulin Resistance. 5-6 November 1997. American Diabetes Association. Diabetes Care 21:310-314

9. Howard G, O'Leary DH, Zaccaro D et al (1996) Insulin sensitivity and atherosclerosis. The Insulin Resistance Atherosclerosis Study (IRAS) Investigators. Circulation 93:1809-1817

10. Zethelius B, Lithell H, Hales CN, Berne C (2005) Insulin sensitivity, proinsulin and insulin as predictors of coronary heart disease. A population-based 10-year, follow-up study in 70-year old men using the euglycaemic insulin clamp. Diabetologia 48:862-867

11. Ferrannini E, Balkau B, Coppack SW et al (2007) Insulin resistance, insulin response, and obesity as indicators of metabolic risk. J Clin Endocrinol Metab 92:2885-2892

12. Ruige JB, Assendelft WJ, Dekker JM, Kostense PJ, Heine RJ, Bouter LM (1998) Insulin and risk of cardiovascular disease: a meta-analysis. Circulation 97:996-1001

13. Hu G, Qiao Q, Tuomilehto J, Eliasson M, Feskens EJ, Pyorala K (2004) Plasma insulin and cardiovascular mortality in nondiabetic European men and women: a meta-analysis of data from eleven prospective studies. Diabetologia 47:1245-1256

14. Gutt M, Davis CL, Spitzer SB et al (2000) Validation of the insulin sensitivity index (ISI $(0,120))$ : comparison with other measures. Diabetes Res Clin Pract 47:177-184

15. Katz A, Nambi SS, Mather K et al (2000) Quantitative insulin sensitivity check index: a simple, accurate method for assessing insulin sensitivity in humans. J Clin Endocrinol Metab 85:2402-2410

16. Matthews DR, Hosker JP, Rudenski AS, Naylor BA, Treacher DF, Turner RC (1985) Homeostasis model assessment: insulin resistance and beta-cell function from fasting plasma glucose and insulin concentrations in man. Diabetologia 28:412-419

17. Wallace TM, Levy JC, Matthews DR (2004) Use and abuse of HOMA modeling. Diabetes Care 27:1487-1495

18. Isomaa B, Almgren P, Tuomi T et al (2001) Cardiovascular morbidity and mortality associated with the metabolic syndrome. Diabetes Care 24:683-689

19. Hedblad B, Nilsson P, Engstrom G, Berglund G, Janzon L (2002) Insulin resistance in non-diabetic subjects is associated with increased incidence of myocardial infarction and death. Diabet Med 19:470-475 
20. Hanley AJ, Williams K, Stern MP, Haffner SM (2002) Homeostasis model assessment of insulin resistance in relation to the incidence of cardiovascular disease: the San Antonio Heart Study. Diabetes Care 25:1177-1184

21. Bonora E, Kiechl S, Willeit J et al (2007) Insulin resistance as estimated by homeostasis model assessment predicts incident symptomatic cardiovascular disease in Caucasian subjects from the general population: the Bruneck study. Diabetes Care 30:318324

22. Rutter MK, Meigs JB, Sullivan LM, D'Agostino RB Sr, Wilson PW (2005) Insulin resistance, the metabolic syndrome, and incident cardiovascular events in the Framingham Offspring Study. Diabetes 54:3252-3257

23. Meigs JB, Rutter MK, Sullivan LM, Fox CS, D'Agostino RB Sr, Wilson PW (2007) Impact of insulin resistance on risk of type 2 diabetes and cardiovascular disease in people with metabolic syndrome. Diabetes Care 30:1219-1225

24. Chien KL, Hsu HC, Su TC, Chen MF, Lee YT, Hu FB (2008) Fasting and postchallenge hyperglycemia and risk of cardiovascular disease in Chinese: the Chin-Shan Community Cardiovascular Cohort study. Am Heart J 156:996-1002

25. Lawlor D, Fraser A, Ebrahim S, Smith G (2007) Independent associations of fasting insulin, glucose, and glycated haemoglobin with stroke and coronary heart disease in older women. PLoS Medicine 4:e263

26. Dunstan DW, Zimmet PZ, Welborn TA et al (2002) The Australian Diabetes, Obesity and Lifestyle Study (AusDiab) methods and response rates. Diabetes Res Clin Pract 57:119129

27. Magliano DJ, Barr EL, Zimmet PZ et al (2008) Glucose indices, health behaviors, and incidence of diabetes in Australia: the Australian Diabetes, Obesity and Lifestyle Study. Diabetes Care 31:267-272

28. Briganti EM, Shaw JE, Chadban SJ et al (2003) Untreated hypertension among Australian adults: the 1999-2000 Australian Diabetes, Obesity and Lifestyle Study (AusDiab). Med J Aust 179:135-139

29. Dalton M, Cameron AJ, Zimmet PZ et al (2003) Waist circumference, waist-hip ratio and body mass index and their correlation with cardiovascular disease risk factors in Australian adults. J Intern Med 254:555-563

30. Magliano D, Liew D, Pater H et al (2003) Accuracy of the Australian National Death Index: comparison with adjudicated fatal outcomes among Australian participants in the Long-term Intervention with Pravastatin in Ischaemic Disease (LIPID) study. Aust N Z J Public Health 27:649-653

31. Barr ELM, Tonkin AM, Welborn TA, Shaw JE (2009) Validity of self-reported cardiovascular disease events in comparison to medical record adjudication and a statewide hospital morbidity database: the AusDiab study. Intern Med J 39:49-53

32. Barr EL, Boyko EJ, Zimmet PZ, Wolfe R, Tonkin AM, Shaw JE (2009) Continuous relationships between non-diabetic hyperglycaemia and both cardiovascular disease and all-cause mortality: the Australian Diabetes, Obesity, and Lifestyle (AusDiab) study. Diabetologia 52:415-424

33. Glantz S, Slinker B (1990) Primer of applied regression and analysis of variance. McGraw-Hill, New York

34. Reaven GM, Laws A (1994) Insulin resistance, compensatory hyperinsulinaemia, and coronary heart disease. Diabetologia 37:948-952

35. Taskinen MR (2005) Type 2 diabetes as a lipid disorder. Curr Mol Med 5:297-308

36. DeFronzo RA (2006) Is insulin resistance atherogenic? Possible mechanisms. Atheroscler Suppl 7:11-15

37. Cameron AJ, Boyko EJ, Sicree RA et al (2008) Central obesity as a precursor to the metabolic syndrome in the AusDiab study and Mauritius. Obesity 16:2707-2716

38. Perry IJ, Wannamethee SG, Whincup PH, Shaper AG, Walker MK, Alberti KG (1996) Serum insulin and incident coronary heart disease in middle-aged British men. Am J Epidemiol 144:224-234

39. Alssema M, Dekker JM, Nijpels G, Stehouwer CD, Bouter LM, Heine RJ (2005) Proinsulin concentration is an independent predictor of all-cause and cardiovascular mortality: an 11-year follow-up of the Hoorn Study. Diabetes Care 28:860-865

40. Eschwege E, Richard JL, Thibult N et al (1985) Coronary heart disease mortality in relation with diabetes, blood glucose and plasma insulin levels. The Paris Prospective Study, ten years later. Horm Metab Res Suppl 15:41-46

41. Welborn TA, Knuiman MW, Ward N, Whittall DE (1994) Serum insulin is a risk marker for coronary heart disease mortality in men but not in women. Diabetes Res Clin Pract 26:51-59

42. Pyorala M, Miettinen H, Laakso M, Pyorala K (2000) Plasma insulin and all-cause, cardiovascular, and noncardiovascular mortality: the 22-year follow-up results of the Helsinki Policemen Study. Diabetes Care 23:1097-1102

43. Metter EJ, Windham BG, Maggio M et al (2008) Glucose and insulin measurements from the oral glucose tolerance test and mortality prediction. Diabetes Care 31:1026-1030

44. Hermans MP, Levy JC, Morris RJ, Turner RC (1999) Comparison of insulin sensitivity tests across a range of glucose tolerance from normal to diabetes. Diabetologia 42:678-687

45. Nilsson P, Nilsson JA, Hedblad B, Eriksson KF, Berglund G (2003) Hyperinsulinaemia as long-term predictor of death and ischaemic heart disease in nondiabetic men: The Malmo Preventive Project. J Intern Med 253:136-145 